\title{
Roles of LncRNAs in Viral Infections
}

\author{
Weiwei Liu and Chan Ding * \\ Avian infectious Department, Shanghai Veterinary Research Institute, Chinese Academy of Agricultural Science, Shanghai, \\ China
}

Many proteins and signaling pathways participate in anti-viral host responses. Long non-coding RNAs (IncRNAs), a subset of non-coding RNAs greater than 200 nucleotides in length, have been recently described as critical regulators in viral infections. Accumulating research indicates that IncRNAs are important in the development and progression of infectious diseases. LncRNAs are not only involved in anti-viral responses, but in many different virus-host interactions, some of which may be beneficial to the virus. Here we review the current knowledge regarding host and viral IncRNAs and their roles in viral infections. In addition, the potential of using IncRNAs as diagnostic biomarkers is discussed.

Keywords: long non-coding RNAs, virus infection, cellular IncRNAs, virus-encoded IncRNAs, cell-virus interaction

\section{INTRODUCTION}

Fewer than $2 \%$ of genes are transcribed into mRNAs. A large number of non-coding RNAs (ncRNAs) also play important cellular functions. Based on their length, ncRNAs can be broadly classified as either short ncRNAs ( $<200$ nucleotides) or long ncRNAs ( $>200$ nucleotides, i.e., lncRNAs). Short ncRNAs can be further classified as small interfering RNAs (siRNAs), microRNAs (miRNAs), and Piwi-interacting RNAs (piRNAs). MiRNAs are the best characterized ncRNAs and are well known to induce mRNA degradation or inhibit mRNA translation via the RNA

OPEN ACCESS

Edited by:

Luka Cicin-Sain,

Helmholtz Zentrum für Infektionsforschung GmbH, Germany

Reviewed by:

Michael Nevels,

University of St Andrews,

United Kingdom

Stephen Noel Waggoner,

Cincinnati Children's Hospital Medical

Center, United States

*Correspondence:

Chan Ding

shoveldeen@shvriac.cn

Received: 08 March 2017 Accepted: 08 May 2017 Published: 26 May 2017

Citation:

Liu W and Ding C (2017) Roles of LncRNAs in Viral Infections.

Front. Cell. Infect. Microbiol. 7:205. doi: 10.3389/fcimb.2017.00205 interference pathway. Compared with miRNA, much less is known about the function of lncRNAs.

LncRNAs are the transcribed and spliced products of RNA polymerase II or III transcription, are $5^{\prime}$ capped, and may contain a polyadenylated tail at the $3^{\prime}$ end. The expression of lncRNAs is much lower in comparison to mRNAs and lncRNAs are expressed in cell-, tissue-, and developmental stage-specific manners (Djebali et al., 2012). According to their position relative to the neighboring protein-coding gene, lncRNAs are classified as sense, antisense, bidirectional, intronic, or intergenic. The human genome encodes thousands of lncRNAs. Previously, lncRNAs were considered as "dark matter" or "junk" in the genome (Doolittle, 2013). However, recent studies have illuminated the roles of lncRNAs, and they are now considered important physiological regulators of cell homeostasis, growth, and differentiation (Wapinski and Chang, 2011; Hu et al., 2012; Fatica and Bozzoni, 2014). Emerging data have also identified the important roles of lncRNAs in regulating anti-viral responses. This review highlights specific lncRNAs associated with viral infection, specifically focusing on their expression and function.

\section{FUNCTIONS AND MECHANISMS}

LncRNAs regulate numerous cellular processes such as gene imprinting, regulation of the p53 pathway, stem cell self-renewal and differentiation, and DNA damage response (Latos et al., 2012; Liu et al., 2013; Yang et al., 2014; Sharma et al., 2015). MiRNAs (about 19-25 nt in length) are known to take part in many of these cellular activities (Ameres and Zamore, 2013; Ha and Kim, 2014). MiRNAs modulate mRNA degradation or translation by basepairing to sequence motifs of mRNAs. In contrast, lncRNAs utilize a multitude of mechanisms, mediated by their specific sequences or structural motifs that bind DNA, RNA, or protein. 
LncRNAs can function in cis to regulate expression of a neighboring gene and in trans to impact gene expression across chromosomes. Furthermore, lncRNAs function as signals, decoys, guides, and scaffolds to regulate different processes, ranging from chromatin remodeling, transcription, to posttranscriptional regulation (Wang and Chang, 2011; Bonasio and Shiekhattar, 2014).

\section{Chromatin Remodeling}

DNA methylation and histone modifications can alter the state of chromatin, resulting in transcriptional activation or silencing. In this setting, lncRNA recruits chromatin remodeling components to specific genomic loci, reprogramming the state of chromatin to silence or activate transcription (Figure 1A). For example, the Hox transcript antisense intergenic RNA (HOTAIR) is an lncRNA expressed from the developmental HOXC locus that can serve as a scaffold to recruit PRC2 and LSD1 in trans, leading to H3K27 methylation and H3K4me2 demethylation. (Gupta et al., 2010; Tsai et al., 2010). H3K27 methylation is associated with transcription repression, while $\mathrm{H} 3 \mathrm{~K} 4 \mathrm{me} 2$ demethylation is associated with transcription activation. Furthermore, lncRNAs can also regulate expression of neighboring genes in cis, especially in imprinting. The lncRNA Air is imprinted and expressed only from the paternal allele, which at the promoter of Slc22a3 recruits G9a and leads to targeted H3K9 methylation and allelic silencing (Nagano et al., 2008).

\section{Transcriptional Regulation}

LncRNAs also act as decoys, signals or guides to play important roles in transcriptional regulation (Figure 1B). Recently, the lncRNA, Lethe, was identified as a pseudogene. Lethe is upregulated directly by NF- $\kappa \mathrm{B}$ after stimulation with TNF- $\alpha$ or glucocorticoid receptor dexamethasone. Furthermore, as a decoy, Lethe can bind to RelA-RelA homodimers and block binding to other NF- $\kappa \mathrm{B}$ response elements, thus inhibiting the function of NF- $\kappa \mathrm{B}$, and leading to decreased expression of downstream effectors, such as IL-6, SOD2, IL-8, and NF-кB (Rapicavoli et al., 2013). Moreover, IncRNA THRIL, and IncRNA-Cox2 regulate the transcription of TNF- $\alpha$ and CCL 5 by binding hnRNP (heterogeneous nuclear ribonucleoprotein) isoforms (Carpenter et al., 2013; Li et al., 2014).

\section{Post-transcriptional Regulation}

LncRNAs also participate in post-transcriptional regulatory networks. LncRNAs have recently been suggested to act as miRNA "sponges" by sharing common miRNA response elements (MREs) and inhibiting normal miRNA targeting activities on mRNA (Figure 1C). Competing endogenous RNAs (ceRNAs) vie with mRNAs for miRNAs with shared MREs and act as modulators of miRNA by influencing the available amount of miRNA(Sen et al., 2014). Linc-MD1 is a cytoplasmic lncRNA expressed during myoblast differentiation that acts as a ceRNA for miR-133 and miR-135 to control MEF2C, MAML1 and myoblast differentiation (Cesana et al., 2011).

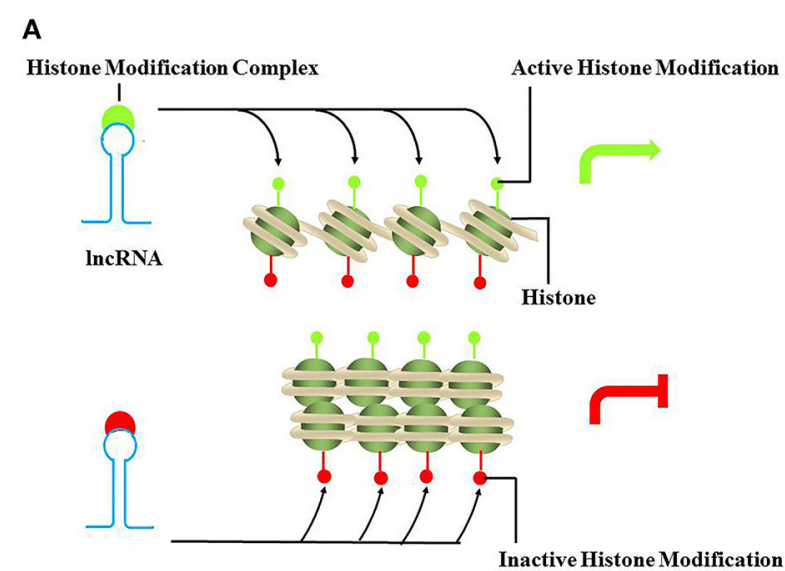

B
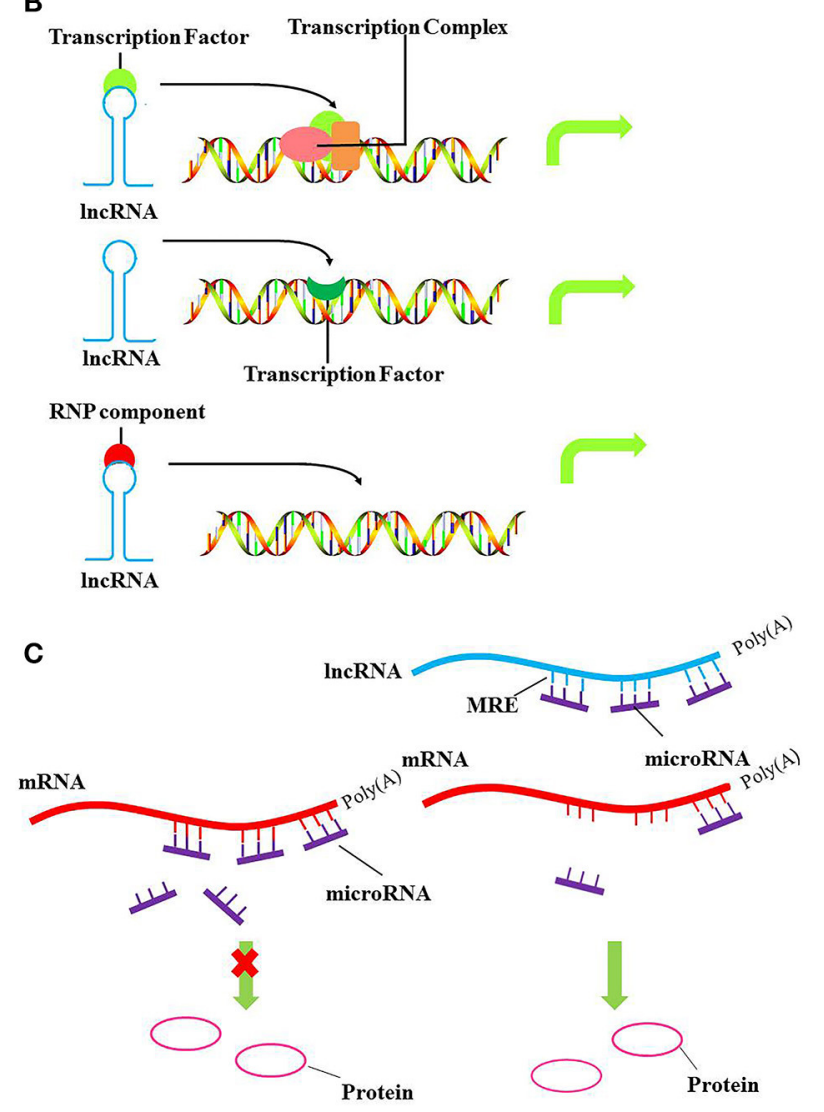

FIGURE 1 | Models of IncRNA mediated chromatin remodeling, transcriptional, and post-transcriptional regulation. (A) LncRNA can act as scaffold to recruit chromatin remodeling components, such as histone modifiers to specific genomic loci and reprogram the state of chromatin to silence or activate transcription. The upper panel and lower panel represent the active and inactive chromatin, respectively. (B) In the upper panel and lower panel, IncRNA can act as decoy or as a guide to bind transcription factors or ribonucleoproteins, altering recruitment to specific genomic loci in cis or in trans, ultimately driving transcription of the localized gene. As shown in the middle panel, IncRNA can also act as signal to regulate gene expression. (C) LncRNAs act as miRNA "sponges" by sharing common MREs, inhibiting normal miRNA targeting activity on mRNA. Green arrows, activate transcription; Red arrows, inhibit transcription. 


\section{LncRNA AND VIRUS INFECTION}

Recently, IncRNAs have been shown to exert both positive and negative effects on innate immunity and virus replication (Ding et al., 2016; Fortes and Morris, 2016). Next, we will discuss the cellular lncRNAs in virus-infected cells, virus-encoded lncRNAs and chimeric lncRNAs formed by viral and cellular sequences, respectively (Table 1).

\section{Cellular LncRNAs in Virus-infected Cells}

Using Next Generation Sequencing (NGS), differential expression of approximately 500 annotated lncRNAs and 1,000 non-annotated genomic regions after SARS coronavirus infection were identified in mice (Peng et al., 2010). This research represented the first discovery of the widespread differential expression of $\operatorname{lncRNAs}$ in response to virus infection and suggested that lncRNAs may be involved in regulating the antiviral host response. Recently, Qi Zhang et al found 646 lncRNAs were upregulated and $424 \operatorname{lncRNAs}$ were downregulated in latent human cytomegalovirus (CMV) infection on THP-1 cells using RNA-seq analysis (Zhang et al., 2016). However, the critical lncRNA in latent human CMV infection has not been identified and its role has not been elucidated yet. Moreover, additional lncRNAs associated with the virus infection in the host have been identified. Significant differential expression of lncRNAs is induced by virus and regulated by RNA virus or DNA virus infection. In turn, these lncRNAs regulate the host innate immune response including the pathogen recognition receptor (PRR)-related signaling, the production of IFNs and cytokines (Carpenter, 2016; Ouyang et al., 2016). For example, in HCV infection, IncRNA-CMPK2 promotes HCV replication and lncRNA-CMPK2 is significantly upregulated in the primary human hepatocytes after treatment with IFN$\alpha$ and knockdown of lncRNA-CMPK2 exhibited a negative regulatory role in the modulation of the IFN response with the increase in the expression of several ISGs, such as Mxl, ISG15 and CXCL10 (Kambara et al., 2014). Recently, Carnero et al found HCV infection increased the expression of lncRNA EGOT, an event that was induced by the NF- $\mathrm{KB}$ activated retinoic acid-inducible gene 1 (RIG-I) and the RNA-activated kinase PKR. Moreover, EGOT expression was also increased after infection with influenza or Semliki Forest virus (SFV) (Carnero et al., 2016). Although, lncRNA EGOT was found to involve in the NF- $\mathrm{KB}$ activated RIG-I and PKR pathway, the specific mechanisms in this pathway and antiviral response remain unclear. Additionally, lncRNA GAS5 was found to be upregulated during HCV infection in Huh7 cells and lncRNA GAS5 inhibited HCV replication by binding viral NS3 protein but the innate immune response remains low (Qian et al., 2016).

In influenza virus infection, Ouyang et al found the expression of the IncRNA NRAV was down regulated after infection with a DNA virus (HSV) and with RNA viruses (SeV and MDRV) (Ouyang et al., 2014). NRAV promotes influenza A virus (IAV) replication and virulence and negatively regulates the expression of several critical IFN-stimulated genes (ISGs), including IFIT2, IFIT3, IFITM3, OASL, and MxA. Among these ISGs, the level of
MxA was most significantly affected by the expression of NRAV and negatively correlated with NRAV expression. NRAV inhibits the initial transcription of MxA and IFITM3 by regulating histone modifications $\mathrm{H} 3 \mathrm{~K} 4 \mathrm{me} 3$ and $\mathrm{H} 3 \mathrm{~K} 27 \mathrm{me} 3$ of the ISG genes. LncBST2/BISPR is expressed from the position in the genome divergent from the well characterized BST2 (a key host cell defense molecule), and lncBST2/BISPR is induced in cells infected with mutants of influenza or VSV. Furthermore, lncBST2/BISPR is upregulated in response to IFN stimulation and was identified as a positive regulator of BST expression. Meanwhile, $\operatorname{lncBST} 2 / \mathrm{BISPR}$ is also induced in cells infected with hepatitis $\mathrm{C}$ virus (HCV) and in the liver of patients with HCV infections (Barriocanal et al., 2015). Although, lncRNAs, NRAV, and $\operatorname{lncBST} 2$, were found in anti-viral response, the specific mechanisms of how they regulate ISG expression have not been elucidated.

LncRNA\#32 is 2,946 nt in length and was identified after poly I: C stimulation. The silencing of IncRNA\#32 remarkably reduced the level of ISG expression, such as IRF7 and OASL, resulting in sensitivity to encephalomyocarditis virus (EMCV) infection. In contrast, overexpression of lncRNA\#32 significantly inhibited EMCV replication. LncRNA\#32 interacts with hnRNPU and ATF2 to regulate ISG expression (Nishitsuji et al., 2016). These results suggested that LncRNA\#32 was involved in anti-viral responses by controlling ISG expression.

In addition to the roles in the antiviral response, lncRNA NEAT1 is necessary for the formation of the nuclear paraspeckles, unique subnuclear structures for the nucleocytoplasmic transport of mRNA in response to certain stimuli (Clemson et al., 2009; Sasaki et al., 2009; Sunwoo et al., 2009; Naganuma and Hirose, 2013). NEAT1, also known as virus-inducible ncRNA (VINC), was first reported in Japanese encephalitis and rabies virus infections of mice (Saha et al., 2006). The expression of NEAT1 was changed by HIV-1 infection and knockdown of NEAT1 enhanced virus production through increased nuclear to cytoplasmic export of Rev-dependent INS-containing HIV1 mRNAs (Zhang et al., 2013). In addition, NEAT1 was also induced by influenza virus and HSV infection, and the expression of antiviral genes including cytokines such as IL-8 was facilitated by cooperative action of NEAT1 and SFPQ (splicing factor proline and glutamine rich, a NEAT1-binding paraspeckle protein) (Imamura et al., 2014).

Theiler's picornavirus is a natural pathogen of mice. A mouse lncRNA, NeST (Nettoie Salmonella pas Theiler's, cleanup Salmonella not Therler's), was identified in Tmevp3 locus on mouse chromosome10 through gene mapping, which is next to the IFN- $\gamma$ coding gene Ifng (Gomez et al., 2013). The transgenic mouse of T cell specific expression of NeST showed that Theiler's virus increased persistence but decreased Salmonella enterica pathogenesis. These observations were likely due to induction of IFN- $\gamma$ transcription specifically in activated CD8+ T cells by NeST. NeST regulates epigenetic marking of the Ifng locus through interaction with a protein partner WDR5, a component of the H3K4 methyltransferase complex. Whether and how disease-associated SNPs alter human NeST expression and/or function has not been elucidated and should be addressed in future studies. 


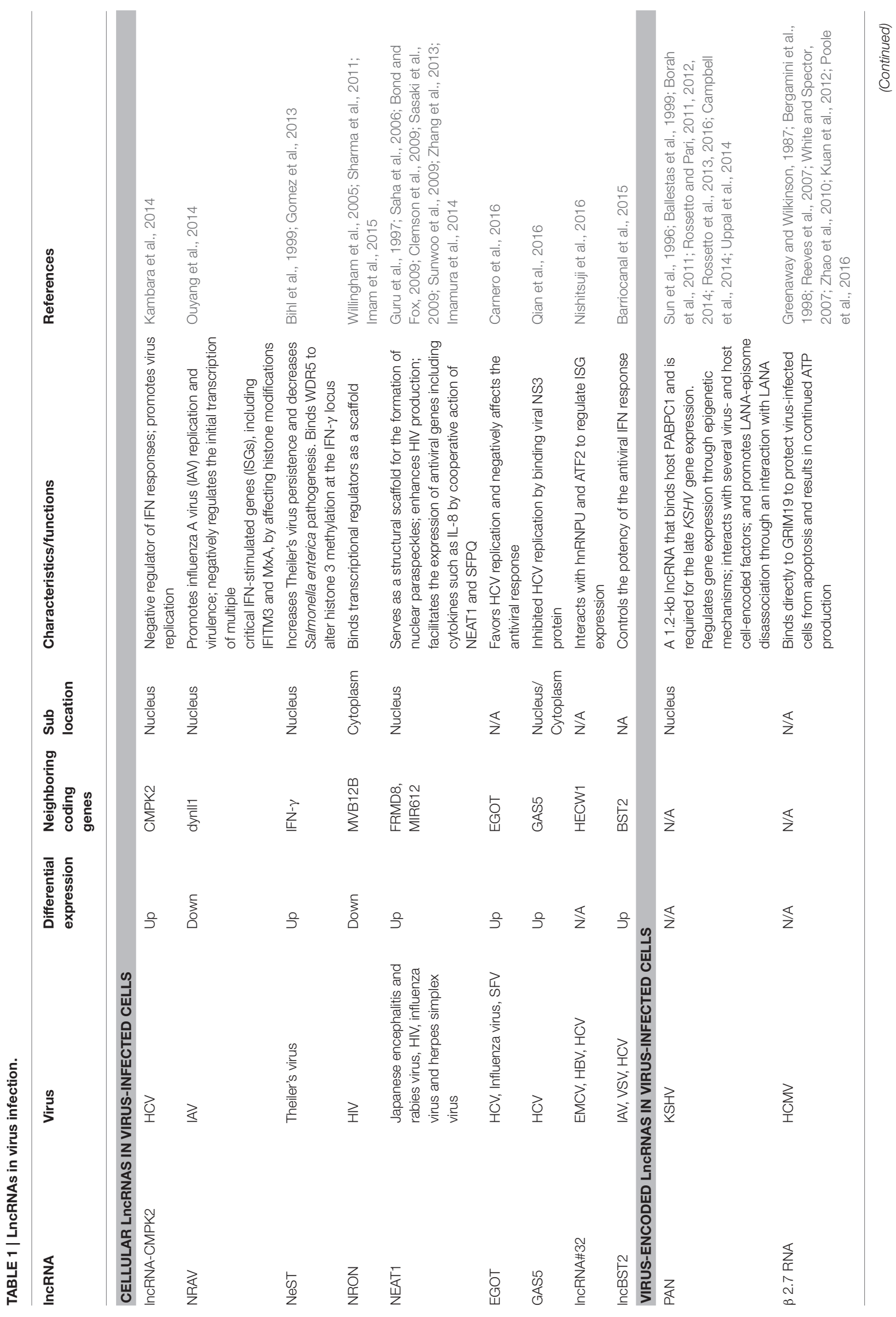


Lit and Ding

LncRNA vs. Virus

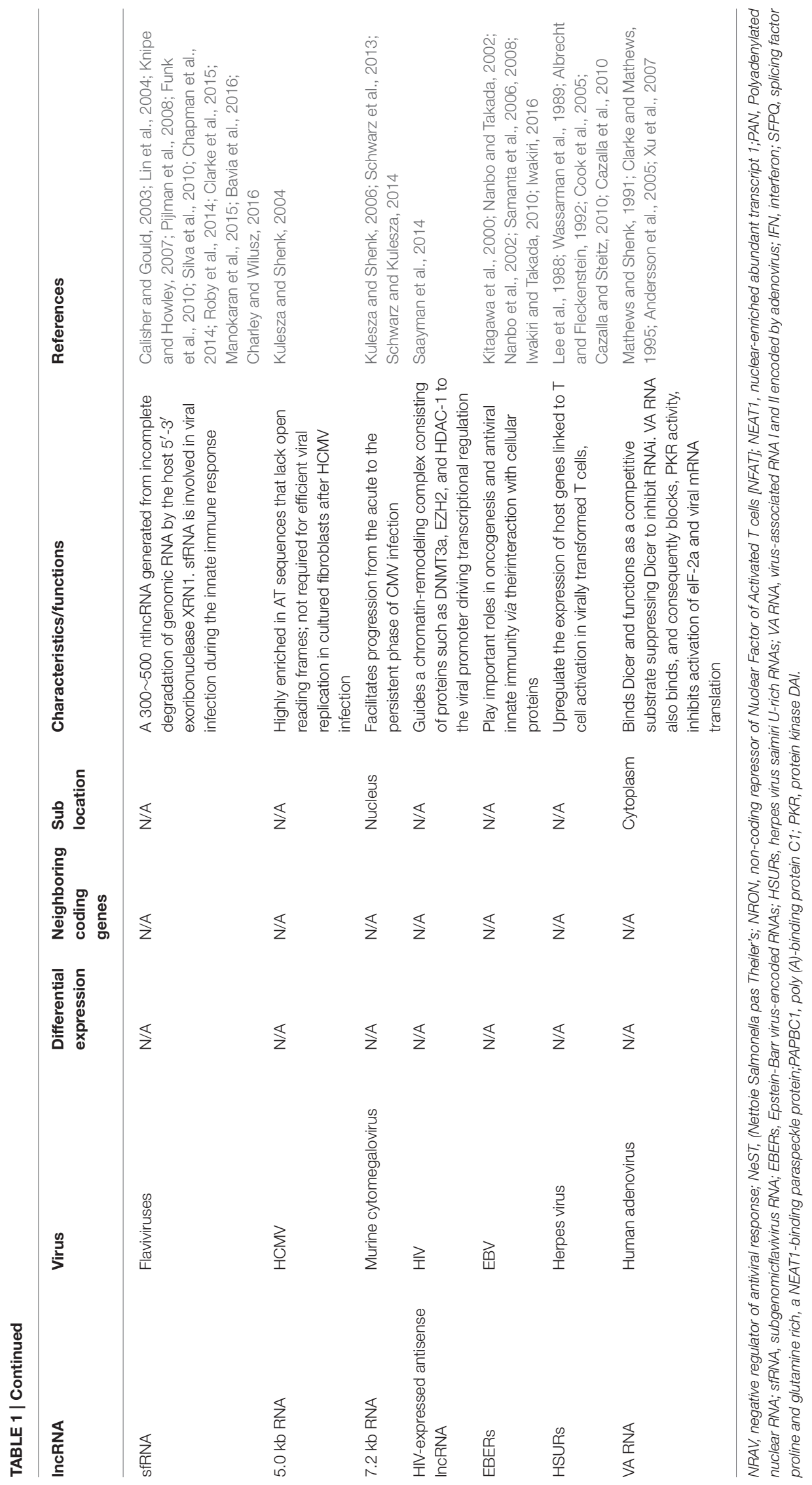

Frontiers in Cellular and Infection Microbiology | www.frontiersin.org

5

May 2017 | Volume 7 | Article 205 
LncRNA NRON (non-coding repressor of NFAT) was initially identified as an inhibitor of transcription factor NFAT (Willingham et al., 2005). NRON interacts with KPNB1, CSE1L, and IQGAP1, which bind phosphorylated NFAT in cytoplasm and represses NFAT nuclear trafficking. When $\mathrm{T}$ cells are activated, dephosphorylated NFAT is released from the complex and enters the nucleus (Sharma et al., 2011). This result suggests that lncRNA exists as a scaffold for a latent transcription factor. A recent study suggested that downregulation of NRON by HIV infection enhanced NFAT nuclear translocation and activity (Imam et al., 2015). HIV also utilizes NRON to control the balance between viral reproduction and cell death through the HIV early expressed protein Nef and the late expressed protein $\mathrm{Vpu}$ to decrease and increase NRON expression at different infection stages respectively (Imam et al., 2015).

\section{Virus-encoded IncRNA}

During virus infection, the host cell generates various $\ln$ cRNAs to counteract infection. Similarly, viruses themselves also express many $\operatorname{lncRNAs}$ to resist cellular antiviral activity. Here, we describe some virus-encoded lncRNAs that have been identified thus far.

Polyadenylated nuclear RNA (PAN) is encoded by Kaposi sarcoma-associated herpes virus (KSHV) and was first identified as a novel abundant $1.2-\mathrm{kb}$ RNA that is transcribed by RNA Polymerase II (Sun et al., 1996). PAN binds host poly (A)binding protein $\mathrm{C} 1$ (PABPC1) after PABPC1 is translocated to the nucleus during the lytic phase of infection and is required for the late KSHV gene expression, such as vIL-6 and k8.1 (Borah et al., 2011). PAN also interacts with the ORF50 promoter and can either repress gene expression by interacting with protein components of polycomb repression complex 2 (PRC2) to mediate the trimethylation of H3K27 or activate gene expression by interacting with UTX, JMJD3 and the histone methyltransferase MLL2 to mediate the removal of the H3K27me3 mark and simultaneously mark it for activation (Rossetto and Pari, 2012; Rossetto et al., 2013, 2016).

In addition, several virus- and host cell-encoded factors, including histones (H1 and $\mathrm{H} 2 \mathrm{~A}$ ), mitochondrial and cellular single-stranded binding proteins (SSBPs) and interferon regulatory factor 4 (IRF4), interact with PAN (Rossetto and Pari, 2011). LANA is essential for maintaining the episomal form of the viral genome during latency (Ballestas et al., 1999; Uppal et al., 2014). PAN promotes LANA-episome disassociation through an interaction with LANA which facilitates LANA sequestration away from KSHV episomes during reactivation (Campbell et al., 2014). Overall, these studies have revealed that PAN as a major global regulator plays an important role in regulation of viral and host gene expression (Rossetto and Pari, 2014).

Recently, transcriptome analysis IncRNA ALT identified lncRNA ALT as an early lytic transcript and a splice isoform of LANA transcript in KSHV infection (Chandriani et al., 2010; Schifano et al., 2017). The size of lncRNA ALT is large (approximately a $\sim 10,000$-nucleotide transcript) and its abundance is very low. However, the specific role of lncRNA ALT remains unclear. $\beta 2.7$ RNA, the most abundantly transcribed early gene from the HCMV genome in permissive cells, is a $2.7-\mathrm{kb}$ unspliced polyadenylated lncRNA (Greenaway and Wilkinson, 1987; White and Spector, 2007). Although, it also has some coding potential, $\beta 2.7$ binds directly to the GRIM19 (genes associated with retinoid/IFN-induced mortality 19), a subunit of mitochondrial enzyme complex I, to protect virus-infected cells from apoptosis and results in continued ATP production, which is critical for the successful completion of the viral life cycle (Bergamini et al., 1998; Reeves et al., 2007). Interaction of the $\beta 2.7$ RNA with complex I inhibits rotenone stress-induced apoptosis in neuronal cells and this suggests that $\beta 2.7$ RNA can be exploited in the development of a novel therapeutic for the treatment of Parkinson's disease (Kuan et al., 2012; Poole et al., 2016). Moreover, $\beta 2.7$ RNA can protect rat aortic endothelial cells from ischemia/reperfusion injury-induced apoptosis by reduction of reactive oxygen species (Zhao et al., 2010).

The subgenomic flavivirus RNA (sfRNA) is $300-500 \mathrm{nt}$ in length and is derived from the $3^{\prime}$ UTR of the RNA genome of flaviviruses, a large group of single-stranded, positive-sense RNA viruses including several human pathogenic viruses, such as yellow fever virus, JEV, dengue viruses, and West Nile virus (Calisher and Gould, 2003; Knipe and Howley, 2007). SfRNA is a product of an incomplete degradation of genomic RNA by the host $5^{\prime}-3^{\prime}$ exoribonuclease XRN1 and sfRNA is involved in viral infection and host cell response modulation (Roby et al., 2014; Clarke et al., 2015; Bavia et al., 2016; Charley and Wilusz, 2016). The rigid secondary structure stem-loop II located at the beginning of the $3^{\prime}$ UTR of the viral genome is resistant to nuclease XRN1 degradation and results in the production of sfRNA (Funk et al., 2010). The sfRNA structure, a ringlike conformation, with the $5^{\prime}$ end of the resistant structure passing through the ring from one side of the fold to the other, is required for the formation sfRNA during flaviviral infection (Chapman et al., 2014). SfRNA generated by the Dengue virus II infection can bind the host proteins G3BP1, G3BP2, and CAPRIN1 and inhibit ISG mRNA translation (Bidet et al., 2014). SfRNA prevents tripartite motif 25 (TRIM25) deubiquitylation, which is critical for sustained and amplified RIG-I-induced type I IFN expression (Manokaran et al., 2015). Production of sfRNA increases the replication efficiency of WNVs and is essential for virus-induced cytotoxicity in cell culture and for viral pathogenicity in mice (Pijlman et al., 2008). However, the mechanisms underlying how sfRNA leads to increased virus replication and cell death remain unknown. SfRNA was also identified in JEV infection and in an RNA pseudoknot that is also necessary for production of yellow fever sfRNA (Lin et al., 2004; Silva et al., 2010).

CMV is a ubiquitous herpes virus that persistently replicates in epithelial cells. A 5-kb immediate-early RNA is a stable intron expressed by human CMV, which is highly AT rich in sequence and lacks open reading frames likely to be translated into protein, and thus is not necessary for efficient replication of the virus in cultured cells after human HCMV infection (Kulesza and Shenk, 2004). A murine CMV 7.2-kb ortholog of the human CMV 5$\mathrm{kb}$ RNA was also identified as a stable intron that facilitates progression from the acute to persistent phase of infection 
(Kulesza and Shenk, 2006). This CMV lncRNA accumulates in the nucleus of infected cells during infection and whose stability is a result of sustained lariat conformation (Schwarz et al., 2013; Schwarz and Kulesza, 2014).

An HIV-encoded antisense lncRNA without a poly (A) tail was recently discovered. This lncRNA guides a chromatinremodeling complex consisting of proteins such as DNMT3a, $\mathrm{EZH} 2$, and HDAC-1 to the viral promoter driving transcriptional regulation (Saayman et al., 2014).

Although, several ncRNAs are $<200$ nt in size, Epstein-Barr virus-encoded RNAs (EBERs), herpes virus saimiri U-rich RNAs (HSURs) and virus-associated RNA I and II (VA I and II) encoded by adenovirus are sometimes also referred to as viral lncRNAs.

Two nuclear, highly structured and abundant viral transcripts EBER1 (167 nt) and EBER2 (172 nt) in latently EBV-infected cells are produced by EBV (Iwakiri, 2016). EBER are polyadenylated, ncRNAs that are transcribed by RNA polymerase III (pol III) (Iwakiri, 2016). EBERs play key roles in antiviral innate immunity via interaction with cellular proteins (Iwakiri and Takada, 2010). EBERs are recognized by RIG-I and activate its downstream signaling to induce expression of type-I IFNs in EBV-infected cells (Samanta et al., 2006). Furthermore, EBERs induce IL10 expression through IRF3, but not NF- $\kappa$ B activation, in BL (Burkitt's lymphoma) cells, suggesting that EBER acts as an autocrine growth factor in BL cells(Kitagawa et al., 2000; Samanta et al., 2008). In addition, EBER also contributes to oncogenesis(Nanbo and Takada, 2002). For example, in BL, EBERs counteract IFN- $\alpha$-induced apoptosis via binding to PKR and inhibition of its phosphorylation (Nanbo et al., 2002).

Herpesvirus saimiri, which causes aggressive T-cell leukemia and lymphoma, encodes 7 HSURs (Herpesvirus saimiri (HVS) U-rich RNAs) (Lee et al., 1988; Wassarman et al., 1989; Albrecht and Fleckenstein, 1992). The expression of host genes linked to T cell activation in virally transformed $\mathrm{T}$ cells was up regulated by HSURs 1 and 2 (Cook et al., 2005). HSUR 1 directs degradation of host mature miR-27 in a sequence-specific and bindingdependent manner in virally transformed $\mathrm{T}$ cells (Cazalla and Steitz, 2010; Cazalla et al., 2010), illustrating a ncRNA to manipulate host-cell gene expression via the miRNA pathway after viral infection.

Two highly structured cytoplasmic RNAs; named VA RNA I and VA RNA II ( 160-170 nts) are produced from RNA polymerase III (pol III) (Mathews and Shenk, 1991). The VA RNAs bind Dicer and function as competitive substrates suppressing Dicer to inhibit the RNAi (Andersson et al., 2005). Notably, compared with VA RNAI, VA RNA II is incorporated into the RNA-induced silencing complex (RISC) (Xu et al., 2007). Adenovirus VA RNA binds PKR and blocks PKR activity, avoiding phosphorylation of eIF-2a and inhibition of viral mRNA translation (Clarke and Mathews, 1995).

\section{REFERENCES}

Albrecht, J. C., and Fleckenstein, B. (1992). Nucleotide sequence of HSUR 6 and HSUR 7, two small RNAs of herpesvirus saimiri. Nucleic Acids Res. 20:1810. doi: $10.1093 / \mathrm{nar} / 20.7 .1810$
In addition to the cellular lncRNAs and virus encoded lncRNAs, HBx-LINE1 was identified as a chimeric lncRNA, which is produced by viral integration into the host genome leading to activation of a LINE-1 sequence such that a chimeric lncRNA is produced (Lau et al., 2014; Moyo et al., 2016). HBxLINE functions as an lncRNA-like RNA in HBV-positive HCC cell lines, which induces the Wnt pathway by increasing the nuclear localization of $\beta$-catenin. So far, it remains unknown whether other chimeric lncRNAs are identified and their roles in the virus infection.

\section{CONCLUSIONS AND PERSPECTIVE}

Although, thousands of lncRNAs are expressed after viral infection, the specific lncRNA with experimentally verified functions is limited, thus the roles and functions of lncRNAs in viral infection require further investigation. A deeper understanding of how the lncRNA transcriptome is altered in the infected cell and how these alterations affect the interaction between the host and virus should also be explored. Such studies may help in the identification novel cellular pathways involved in the antivirus response.

In addition to the role of lncRNA associated with the antivirus response, lncRNAs may be both unique diagnostic biomarkers as well as novel targets against which new therapeutics can be developed. Virus-related lncRNAs secreted into the serum may serve as prognostic markers. For example, two serum lncRNAs, uc001ncr and AX800134, have potential as novel potential biomarkers to diagnose HBV-positive HCC, especially in the early stage of disease (Wang et al., 2015). The expression of IncRNA-UCA1 and lncRNA-WRAP53 were significantly higher in sera of HCC than in chronic HCV infection or healthy volunteers (Kamel et al., 2016). This result suggests that IncRNA-UCA1 and IncRNA-WRAP53 upregulation may serve as novel serum biomarkers for HCC diagnosis and prognosis. In conclusion, lncRNAs are key regulators of transcriptional and post-transcriptional processes; thus, their roles in virus infection and therapy necessitate intensive study in the future.

\section{AUTHOR CONTRIBUTIONS}

WL wrote this manuscript and CD designed this project.

\section{ACKNOWLEDGMENTS}

This study was supported by the Agricultural Science and Technology Innovation Program (ASTIP) of Chinese Academy of Agricultural Science. We thank Accdon for their linguistic assistance during the preparation of this manuscript.

Ameres, S. L., and Zamore, P. D. (2013). Diversifying microRNA sequence and function. Nat. Rev. Mol. Cell Biol. 14, 475-488. doi: 10.1038/nrm3611

Andersson, M. G., Haasnoot, P. C., Xu, N., Berenjian, S., Berkhout, B., and Akusjarvi, G. (2005). Suppression of RNA interference by adenovirus virusassociated RNA. J. Virol. 79, 9556-9565. doi: 10.1128/JVI.79.15.9556-9565.2005 
Ballestas, M. E., Chatis, P. A., and Kaye, K. M. (1999). Efficient persistence of extrachromosomal KSHV DNA mediated by latency-associated nuclear antigen. Science 284, 641-644. doi: 10.1126/science.284.5414.641

Barriocanal, M., Carnero, E., Segura, V., and Fortes, P. (2015). Long non-coding RNA BST2/BISPR is induced by IFN and regulates the expression of the antiviral factor tetherin. Front. Immunol. 5:655. doi: 10.3389/fimmu.2014.00655

Bavia, L., Mosimann, A. L., Aoki, M. N., and Duarte Dos Santos, C. N. (2016). A glance at subgenomic flavivirus RNAs and microRNAs in flavivirus infections. Virol. J. 13:84. doi: 10.1186/s12985-016-0541-3

Bergamini, G., Reschke, M., Battista, M. C., Boccuni, M. C., Campanini, F., Ripalti, A., et al. (1998). The major open reading frame of the $\beta 2.7$ transcript of human cytomegalovirus: in vitro expression of a protein posttranscriptionally regulated by the $5^{\prime}$ region. J. Virol. 72, 8425-8429.

Bidet, K., Dadlani, D., and Garcia-Blanco, M. A. (2014). G3BP1, G3BP2 and CAPRIN1 are required for translation of interferon stimulated mRNAs and are targeted by a dengue virus non-coding RNA. PLoS Pathog. 10:e1004242. doi: 10.1371/journal.ppat.1004242

Bihl, F., Brahic, M., and Bureau, J. F. (1999). Two loci, Tmevp2 and Tmevp3, located on the telomeric region of chromosome 10, control the persistence of Theiler's virus in the central nervous system of mice. Genetics 152, 385-392.

Bonasio, R., and Shiekhattar, R. (2014). Regulation of transcription by long noncoding RNAs. Annu. Rev. Genet. 48, 433-455. doi: 10.1146/annurev-genet-120213-092323

Bond, C. S., and Fox, A. H. (2009). Paraspeckles: nuclear bodies built on long noncoding RNA. J. Cell Biol. 186, 637-644. doi: 10.1083/jcb.200906113

Borah, S., Darricarrere, N., Darnell, A., Myoung, J., and Steitz, J. A. (2011). A viral nuclear noncoding RNA binds re-localized poly(A) binding protein and is required for late KSHV gene expression. PLoS Pathog. 7:e1002300. doi: 10.1371 /journal.ppat.1002300

Calisher, C. H., and Gould, E. A. (2003). Taxonomy of the virus family Flaviviridae. Adv. Virus Res. 59, 1-19. doi: 10.1016/S0065-3527(03)59001-7

Campbell, M., Kim, K. Y., Chang, P. C., Huerta, S., Shevchenko, B., Wang, D. H., et al. (2014). A lytic viral long noncoding RNA modulates the function of a latent protein. J. Virol. 88, 1843-1848. doi: 10.1128/JVI.03251-13

Carnero, E., Barriocanal, M., Prior, C., Pablo Unfried, J., Segura, V., Guruceaga, E., et al. (2016). Long noncoding RNA EGOT negatively affects the antiviral response and favors HCV replication EMBO Rep. 17, 1013-1028. doi: 10.15252/embr.201541763

Carpenter, S. (2016). Long noncoding RNA: novel links between gene expression and innate immunity. Virus Res. 212, 137-145. doi: 10.1016/j.virusres.2015.08.019

Carpenter, S., Aiello, D., Atianand, M. K., Ricci, E. P., Gandhi, P., Hall, L. L., et al. (2013). A long noncoding RNA mediates both activation and repression of immune response genes. Science 341, 789-792. doi: 10.1126/science.1240925

Cazalla, D., and Steitz, J. A. (2010). Down-regulation of a host microRNA by a viral noncoding RNA. Cold Spring Harb. Symp. Quant. Biol. 75, 321-324. doi: $10.1101 / \mathrm{sqb} .2010 .75 .009$

Cazalla, D., Yario, T., and Steitz, J. A. (2010). Down-regulation of a host microRNA by a Herpesvirus saimiri noncoding RNA. Science 328, 1563-1566. doi: $10.1126 /$ science. 1187197

Cesana, M., Cacchiarelli, D., Legnini, I., Santini, T., Sthandier, O., Chinappi, M., et al. (2011). A long noncoding RNA controls muscle differentiation by functioning as a competing endogenous RNA. Cell 147, 358-369. doi: 10.1016/j.cell.2011.09.028

Chandriani, S., Xu, Y., and Ganem, D. (2010). The lytic transcriptome of Kaposi's sarcoma-associated herpesvirus reveals extensive transcription of noncoding regions, including regions antisense to important genes. J. Virol. 84, 7934-7942. doi: 10.1128/JVI.00645-10

Chapman, E. G., Costantino, D. A., Rabe, J. L., Moon, S. L., Wilusz, J., Nix, J. C., et al. (2014). The structural basis of pathogenic subgenomic flavivirus RNA (sfRNA) production. Science 344, 307-310. doi: 10.1126/science.1250897

Charley, P. A., and Wilusz, J. (2016). Standing your ground to exoribonucleases: function of Flavivirus long non-coding RNAs. Virus Res. 212, 70-77. doi: 10.1016/j.virusres.2015.09.009

Clarke, B. D., Roby, J. A., Slonchak, A., and Khromykh, A. A. (2015). Functional non-coding RNAs derived from the flavivirus $3^{\prime}$ untranslated region. Virus Res. 206, 53-61. doi: 10.1016/j.virusres.2015.01.026
Clarke, P., and Mathews, M. (1995). Interactions between the double-stranded RNA binding motif and RNA: definition of the binding site for the interferoninduced protein kinase DAI (PKR) on adenovirus VA RNA. RNA 1, 7-20.

Clemson, C. M., Hutchinson, J. N., Sara, S. A., Ensminger, A. W., Fox, A. H., Chess, A., et al. (2009). An architectural role for a nuclear noncoding RNA: NEAT1 RNA is essential for the structure of paraspeckles. Mol. Cell 33, 717-726. doi: 10.1016/j.molcel.2009.01.026

Cook, H. L., Lytle, J. R., Mischo, H. E., Li, M. J., Rossi, J. J., Silva, D. P., et al. (2005). Small nuclear RNAs encoded by Herpesvirus saimiri upregulate the expression of genes linked to $\mathrm{T}$ cell activation in virally transformed T cells. Curr. Biol. 15, 974-979. doi: 10.1016/j.cub.2005.04.034

Ding, Y. Z., Zhang, Z. W., Liu, Y. L., Shi, C. X., Zhang, J., and Zhang, Y. G. (2016). Relationship of long noncoding RNA and viruses. Genomics 107, 150-154. doi: 10.1016/j.ygeno.2016.01.007

Djebali, S., Davis, C. A., Merkel, A., Dobin, A., Lassmann, T., Mortazavi, A., et al. (2012). Landscape of transcription in human cells. Nature 489, 101-108. doi: 10.1038/nature11233

Doolittle, W. F. (2013). Is junk DNA bunk? A critique of ENCODE. Proc. Natl. Acad. Sci. U.S.A. 110, 5294-5300. doi: 10.1073/pnas.1221376110

Fatica, A., and Bozzoni, I. (2014). Long non-coding RNAs: new players in cell differentiation and development. Nat. Rev. Genet. 15, 7-21. doi: $10.1038 / \mathrm{nrg} 3606$

Fortes, P., and Morris, K. V. (2016). Long noncoding RNAs in viral infections. Virus Res. 212, 1-11. doi: 10.1016/j.virusres.2015.10.002

Funk, A., Truong, K., Nagasaki, T., Torres, S., Floden, N., Balmori Melian, E., et al. (2010). RNA structures required for production of subgenomic flavivirus RNA. J. Virol. 84, 11407-11417. doi: 10.1128/JVI.01159-10

Gomez, J. A., Wapinski, O. L., Yang, Y. W., Bureau, J. F., Gopinath, S., Monack, D. M., et al. (2013). The NeST long ncRNA controls microbial susceptibility and epigenetic activation of the interferon-gamma locus. Cell 152, 743-754. doi: 10.1016/j.cell.2013.01.015

Greenaway, P. J., and Wilkinson, G. W. (1987). Nucleotide sequence of the most abundantly transcribed early gene of human cytomegalovirus strain AD169. Virus Res. 7, 17-31. doi: 10.1016/0168-1702(87)90055-4

Gupta, R. A., Shah, N., Wang, K. C., Kim, J., Horlings, H. M., Wong, D. J., et al. (2010). Long non-coding RNA HOTAIR reprograms chromatin state to promote cancer metastasis. Nature 464, 1071-1076. doi: 10.1038/nature 08975

Guru, S. C., Agarwal, S. K., Manickam, P., Olufemi, S. E., Crabtree, J. S., Weisemann, J. M., et al. (1997). A transcript map for the 2.8-Mb region containing the multiple endocrine neoplasia type 1 locus. Genome Res. 7, 725-735.

Ha, M., and Kim, V. N. (2014). Regulation of microRNA biogenesis. Nat. Rev. Mol. Cell Biol. 15, 509-524. doi: 10.1038/nrm3838

Hu, W., Alvarez-Dominguez, J. R., and Lodish, H. F. (2012). Regulation of mammalian cell differentiation by long non-coding RNAs. EMBO Rep. 13, 971-983. doi: 10.1038/embor.2012.145

Imam, H., Bano, A. S., Patel, P., Holla, P., and Jameel, S. (2015). The IncRNA NRON modulates HIV-1 replication in a NFAT-dependent manner and is differentially regulated by early and late viral proteins. Sci. Rep. 5:8639. doi: 10.1038/srep08639

Imamura, K., Imamachi, N., Akizuki, G., Kumakura, M., Kawaguchi, A., Nagata, K., et al. (2014). Long noncoding RNA NEAT1-dependent SFPQ relocation from promoter region to paraspeckle mediates IL8 expression upon immune stimuli. Mol. Cell 53, 393-406. doi: 10.1016/j.molcel.2014.01.009

Iwakiri, D. (2016). Multifunctional non-coding Epstein-Barr virus encoded RNAs (EBERs) contribute to viral pathogenesis. Virus Res. 212, 30-38. doi: 10.1016/j.virusres.2015.08.007

Iwakiri, D., and Takada, K. (2010). Role of EBERs in the pathogenesis of EBV infection. Adv. Cancer Res. 107, 119-136. doi: 10.1016/S0065-230X(10)07004-1

Kambara, H., Niazi, F., Kostadinova, L., Moonka, D. K., Siegel, C. T., Post, A. B., et al. (2014). Negative regulation of the interferon response by an interferon-induced long non-coding RNA. Nucleic Acids Res. 42, 10668-10680. doi: $10.1093 /$ nar/gku713

Kamel, M. M., Matboli, M., Sallam, M., Montasser, I. F., Saad, A. S., and El-Tawdi, A. H. (2016). Investigation of long noncoding RNAs expression profile as potential serum biomarkers in patients with hepatocellular carcinoma. Transl. Res. 168, 134-145. doi: 10.1016/j.trsl.2015.10.002 
Kitagawa, N., Goto, M., Kurozumi, K., Maruo, S., Fukayama, M., Naoe, T., et al. (2000). Epstein-Barr virus-encoded poly(A)(-) RNA supports Burkitt's lymphoma growth through interleukin-10 induction. EMBO J. 19, 6742-6750. doi: $10.1093 / \mathrm{emboj} / 19.24 .6742$

Knipe, D. M., and Howley, P. M. (2007). Flaviviridae: The Viruses and Their Replication. Fields Virology, 5th Edn. Philadelphia, PA: Lippincott-Raven Publishers.

Kuan, W. L., Poole, E., Fletcher, M., Karniely, S., Tyers, P., Wills, M., et al. (2012). A novel neuroprotective therapy for Parkinson's disease using a viral noncoding RNA that protects mitochondrial complex I activity. J. Exp. Med. 209, 1-10. doi: 10.1084/jem.20111126

Kulesza, C. A., and Shenk, T. (2004). Human cytomegalovirus 5-kilobase immediate-early RNA is a stable intron. J. Virol. 78, 13182-13189. doi: 10.1128/JVI.78.23.13182-13189.2004

Kulesza, C. A., and Shenk, T. (2006). Murine cytomegalovirus encodes a stable intron that facilitates persistent replication in the mouse. Proc. Natl. Acad. Sci. U.S.A. 103, 18302-18307. doi: 10.1073/pnas.0608718103

Latos, P. A., Pauler, F. M., Koerner, M. V., Senergin, H. B., Hudson, Q. J., Stocsits, R. R., et al. (2012). Airn transcriptional overlap, but not its IncRNA products, induces imprinted Igf2r silencing. Science 338, 1469-1472. doi: $10.1126 /$ science. 1228110

Lau, C. C., Sun, T., Ching, A. K., He, M., Li, J. W., Wong, A. M., et al. (2014). Viral-human chimeric transcript predisposes risk to liver cancer development and progression. Cancer Cell 25, 335-349. doi: 10.1016/j.ccr.2014.01.030

Lee, S. I., Murthy, S. C., Trimble, J. J., Desrosiers, R. C., and Steitz, J. A. (1988). Four novel U RNAs are encoded by a herpesvirus. Cell 54, 599-607. doi: 10.1016/S0092-8674(88)80004-7

Li, Z., Chao, T. C., Chang, K. Y., Lin, N., Patil, V. S., Shimizu, C., et al. (2014). The long noncoding RNA THRIL regulates TNF $\alpha$ expression through its interaction with hnRNPL. Proc. Natl. Acad. Sci. U.S.A. 111, 1002-1007. doi: 10.1073/pnas.1313768111

Lin, K. C., Chang, H. L., and Chang, R. Y. (2004). Accumulation of a $3^{\prime}$-terminal genome fragment in Japanese encephalitis virus-infected mammalian and mosquito cells. J. Virol. 78, 5133-5138. doi: 10.1128/JVI.78.10.5133-5138.2004

Liu, Q., Huang, J., Zhou, N., Zhang, Z., Zhang, A., Lu, Z., et al. (2013). LncRNA loc285194 is a p53-regulated tumor suppressor. Nucleic Acids Res. 41, 4976-4987. doi: 10.1093/nar/gkt182

Manokaran, G., Finol, E., Wang, C., Gunaratne, J., Bahl, J., Ong, E. Z., et al. (2015). Dengue subgenomic RNA binds TRIM25 to inhibit interferon expression for epidemiological fitness. Science 350, 217-221. doi: 10.1126/science.aab3369

Mathews, M. B., and Shenk, T. (1991). Adenovirus virus-associated RNA and translation control. J. Virol. 65, 5657-5662.

Moyo, B., Nicholson, S. A., and Arbuthnot, P. B. (2016). The role of long noncoding RNAs in hepatitis B virus-related hepatocellular carcinoma. Virus Res. 212, 103-113. doi: 10.1016/j.virusres.2015.07.025

Nagano, T., Mitchell, J. A., Sanz, L. A., Pauler, F. M., Ferguson-Smith, A. C., Feil, R., et al. (2008). The Air noncoding RNA epigenetically silences transcription by targeting G9a to chromatin. Science 322, 1717-1720. doi: $10.1126 /$ science. 1163802

Naganuma, T., and Hirose, T. (2013). Paraspeckle formation during the biogenesis of long non-coding RNAs. RNA Biol. 10, 456-461. doi: 10.4161/rna.23547

Nanbo, A., Inoue, K., Adachi-Takasawa, K., and Takada, K. (2002). Epstein-Barr virus RNA confers resistance to interferon- $\alpha$-induced apoptosis in Burkitt's lymphoma. EMBO J. 21, 954-965. doi: 10.1093/emboj/21.5.954

Nanbo, A., and Takada, K. (2002). The role of Epstein-Barr virus-encoded small RNAs (EBERs) in oncogenesis. Rev. Med. Virol. 12, 321-326. doi: $10.1002 /$ rmv.363

Nishitsuji, H., Ujino, S., Yoshio, S., Sugiyama, M., Mizokami, M., Kanto, T., and Shimotohno, K. (2016). Long noncoding RNA \#32 contributes to antiviral responses by controlling interferon-stimulated gene expression. Proc. Natl. Acad. Sci. 113, 10388-10393.

Ouyang, J., Hu, J., and Chen, J. L. (2016). IncRNAs regulate the innate immune response to viral infection. Wiley Interdiscip. Rev. RNA 7, 129-143. doi: 10.1002/wrna.1321

Ouyang, J., Zhu, X., Chen, Y., Wei, H., Chen, Q., Chi, X., et al. (2014). NRAV, a long noncoding RNA, modulates antiviral responses through suppression of interferon-stimulated gene transcription. Cell Host Microbe 16, 616-626. doi: 10.1016/j.chom.2014.10.001
Peng, X., Gralinski, L., Armour, C. D., Ferris, M. T., Thomas, M. J., Proll, S., et al. (2010). Unique signatures of long noncoding RNA expression in response to virus infection and altered innate immune signaling. MBio 1:e00206-10. doi: $10.1128 / \mathrm{mBio} .00206-10$

Pijlman, G. P., Funk, A., Kondratieva, N., Leung, J., Torres, S., Van Der Aa, L., et al. (2008). A highly structured, nuclease-resistant, noncoding RNA produced by flaviviruses is required for pathogenicity. Cell Host Microbe 4, 579-591. doi: 10.1016/j.chom.2008.10.007

Poole, E., Kuan, W. L., Barker, R., and Sinclair, J. (2016). The human cytomegalovirus non-coding Beta2.7 RNA as a novel therapeutic for Parkinson's disease-Translational research with no translation. Virus Res. 212, 64-69. doi: 10.1016/j.virusres.2015.05.007

Qian, X., Xu, C., Zhao, P., and Qi, Z. (2016). Long non-coding RNA GAS5 inhibited hepatitis $\mathrm{C}$ virus replication by binding viral NS3 protein. Virology 492, 155-165. doi: 10.1016/j.virol.2016.02.020

Rapicavoli, N. A., Qu, K., Zhang, J., Mikhail, M., Laberge, R. M., and Chang, H. Y. (2013). A mammalian pseudogene lncRNA at the interface of inflammation and anti-inflammatory therapeutics. Elife 2:e00762. doi: 10.7554/eLife.00762

Reeves, M. B., Davies, A. A., McSharry, B. P., Wilkinson, G. W., and Sinclair, J. H. (2007). Complex I binding by a virally encoded RNA regulates mitochondriainduced cell death. Science 316, 1345-1348. doi: 10.1126/science.1142984

Roby, J. A., Pijlman, G. P., Wilusz, J., and Khromykh, A. A. (2014). Noncoding subgenomic flavivirus RNA: multiple functions in West Nile virus pathogenesis and modulation of host responses. Viruses 6, 404-427. doi: 10.3390/v6020404

Rossetto, C. C., and Pari, G. (2012). KSHV PAN RNA associates with demethylases UTX and JMJD3 to activate lytic replication through a physical interaction with the virus genome. PLoS Pathog. 8:e1002680. doi: 10.1371/journal.ppat.1002680

Rossetto, C. C., and Pari, G. S. (2011). Kaposi's sarcoma-associated herpesvirus noncoding polyadenylated nuclear RNA interacts with virus- and host cellencoded proteins and suppresses expression of genes involved in immune modulation. J. Virol. 85, 13290-13297. doi: 10.1128/JVI.05886-11

Rossetto, C. C., and Pari, G. S. (2014). PAN's labyrinth: molecular biology of Kaposi's Sarcoma-Associated Herpesvirus (KSHV) PAN RNA, a multifunctional long noncoding RNA. Viruses 6, 4212-4226. doi: $10.3390 / v 6114212$

Rossetto, C. C., Tarrant-Elorza, M., Verma, S., Purushothaman, P., and Pari, G. S. (2013). Regulation of viral and cellular gene expression by Kaposi's sarcomaassociated herpesvirus polyadenylated nuclear RNA. J. Virol. 87, 5540-5553. doi: 10.1128/JVI.03111-12

Rossetto, C. C., Tarrant-Elorza, M., Verma, S., Purushothaman, P., and Pari, G. S. (2016). Correction for Rossetto et al., regulation of viral and cellular gene expression by Kaposi's Sarcoma-Associated Herpesvirus polyadenylated nuclear RNA. J. Virol. 90:4255. doi: 10.1128/JVI.00171-16

Saayman, S., Ackley, A., Turner, A. M., Famiglietti, M., Bosque, A., Clemson, M., et al. (2014). An HIV-encoded antisense long noncoding RNA epigenetically regulates viral transcription. Mol. Ther. 22, 1164-1175. doi: 10.1038/mt.2014.29

Saha, S., Murthy, S., and Rangarajan, P. N. (2006). Identification and characterization of a virus-inducible non-coding RNA in mouse brain. J. Gen. Virol. 87, 1991-1995. doi: 10.1099/vir.0.81768-0

Samanta, M., Iwakiri, D., Kanda, T., Imaizumi, T., and Takada, K. (2006). EB virusencoded RNAs are recognized by RIG-I and activate signaling to induce type I IFN. EMBO J. 25, 4207-4214. doi: 10.1038/sj.emboj.7601314

Samanta, M., Iwakiri, D., and Takada, K. (2008). Epstein-Barr virus-encoded small RNA induces IL-10 through RIG-I-mediated IRF-3 signaling. Oncogene 27, 4150-4160. doi: 10.1038/onc.2008.75

Sasaki, Y. T., Ideue, T., Sano, M., Mituyama, T., and Hirose, T. (2009). MEN $\varepsilon / \beta$ noncoding RNAs are essential for structural integrity of nuclear paraspeckles. Proc. Natl. Acad. Sci. U.S.A. 106, 2525-2530. doi: 10.1073/pnas.0807 899106

Schifano, J. M., Corcoran, K., Kelkar, H., and Dittmer, D. P. (2017). Expression of the antisense-to-latency transcript long noncoding RNA in Kaposi's SarcomaAssociated Herpesvirus. J. Virol. 91:e01698-16. doi: 10.1128/JVI.01698-16

Schwarz, T. M., and Kulesza, C. A. (2014). Stability determinants of murine cytomegalovirus long noncoding RNA7.2. J. Virol. 88, 11630-11633. doi: 10.1128/JVI.01695-14

Schwarz, T. M., Volpe, L. A., Abraham, C. G., and Kulesza, C. A. (2013). Molecular investigation of the $7.2 \mathrm{~kb}$ RNA of murine cytomegalovirus. Virol. J. 10:348. doi: $10.1186 / 1743-422 \mathrm{X}-10-348$ 
Sen, R., Ghosal, S., Das, S., Balti, S., and Chakrabarti, J. (2014). Competing endogenous RNA: the key to posttranscriptional regulation. Sci. World J. 2014:896206. doi: 10.1155/2014/896206

Sharma, S., Findlay, G. M., Bandukwala, H. S., Oberdoerffer, S., Baust, B., Li, Z., et al. (2011). Dephosphorylation of the nuclear factor of activated T cells (NFAT) transcription factor is regulated by an RNA-protein scaffold complex. Proc. Natl. Acad. Sci. U.S.A. 108, 11381-11386. doi: 10.1073/pnas.1019711108

Sharma, V., Khurana, S., Kubben, N., Abdelmohsen, K., Oberdoerffer, P., Gorospe, M., et al. (2015). A BRCA1-interacting lncRNA regulates homologous recombination. EMBO Rep. 16, 1520-1534. doi: 10.15252/embr.201540437

Silva, P. A., Pereira, C. F., Dalebout, T. J., Spaan, W. J., and Bredenbeek, P. J. (2010). An RNA pseudoknot is required for production of yellow fever virus subgenomic RNA by the host nuclease XRN1. J. Virol. 84, 11395-11406. doi: 10.1128/JVI.01047-10

Sun, R., Lin, S. F., Gradoville, L., and Miller, G. (1996). Polyadenylylated nuclear RNA encoded by Kaposi sarcoma-associated herpesvirus. Proc. Natl. Acad. Sci. U.S.A. 93, 11883-11888. doi: 10.1073/pnas.93.21.11883

Sunwoo, H., Dinger, M. E., Wilusz, J. E., Amaral, P. P., Mattick, J. S., and Spector, D. L. (2009). MEN $\varepsilon / \beta$ nuclear-retained non-coding RNAs are up-regulated upon muscle differentiation and are essential components of paraspeckles. Genome Res. 19, 347-359. doi: 10.1101/gr.087775.108

Tsai, M. C., Manor, O., Wan, Y., Mosammaparast, N., Wang, J. K., Lan, F., et al. (2010). Long noncoding RNA as modular scaffold of histone modification complexes. Science 329, 689-693. doi: 10.1126/science.1192002

Uppal, T., Banerjee, S., Sun, Z., Verma, S. C., and Robertson, E. S. (2014). KSHV LANA-the master regulator of KSHV latency. Viruses 6, 4961-4998. doi: $10.3390 / \mathrm{v} 6124961$

Wang, K. C., and Chang, H. Y. (2011). Molecular mechanisms of long noncoding RNAs. Mol. Cell 43, 904-914. doi: 10.1016/j.molcel.2011.08.018

Wang, K., Guo, W. X., Li, N., Gao, C. F., Shi, J., Tang, Y. F., et al. (2015). Serum LncRNAs profiles serve as novel potential biomarkers for the diagnosis of HBV-positive hepatocellular carcinoma. PLOS ONE 10:e144934. doi: 10.1371/journal.pone.0144934

Wapinski, O., and Chang, H. Y. (2011). Long noncoding RNAs and human disease. Trends Cell Biol. 21, 354-361. doi: 10.1016/j.tcb.2011.04.001

Wassarman, D. A., Lee, S. I., and Steitz, J. A. (1989). Nucleotide sequence of HSUR 5 RNA from herpesvirus saimiri. Nucleic Acids Res. 17:1258. doi: $10.1093 /$ nar/17.3.1258
White, E. A., and Spector, D. H. (2007). "Early viral gene expression and function," in Human Herpesviruses: Biology, Therapy, and Immunoprophylaxis, eds A. Arvin, G. Campadelli-Fiume, E. Mocarski, P. S. Moore, B. Roizman, R. Whitley, and K. Yamanishi. (Cambridge: Cambridge University Press), 264-294.

Willingham, A. T., Orth, A. P., Batalov, S., Peters, E. C., Wen, B. G., AzaBlanc, P., et al. (2005). A strategy for probing the function of noncoding RNAs finds a repressor of NFAT. Science 309, 1570-1573. doi: 10.1126/science. 1115901

Xu, N., Segerman, B., Zhou, X., and Akusjarvi, G. (2007). Adenovirus virusassociated RNAII-derived small RNAs are efficiently incorporated into the rna-induced silencing complex and associate with polyribosomes. J. Virol. 81, 10540-10549. doi: 10.1128/JVI.00885-07

Yang, Y. W., Flynn, R. A., Chen, Y., Qu, K., Wan, B., Wang, K. C., et al. (2014). Essential role of lncRNA binding for WDR5 maintenance of active chromatin and embryonic stem cell pluripotency. Elife 3:e02046. doi: 10.7554/eLife. 02046

Zhang, Q., Chen, C. Y., Yedavalli, V. S., and Jeang, K. T. (2013). NEAT1 long noncoding RNA and paraspeckle bodies modulate HIV-1 posttranscriptional expression. MBio 4, e00596-e00512. doi: 10.1128/mBio.00596-12

Zhang, Q., Lai, M. M., Lou, Y. Y., Guo, B. H., Wang, H. Y., and Zheng, X. Q. (2016). Transcriptome altered by latent human cytomegalovirus infection on THP-1 cells using RNA-seq. Gene 594, 144-150. doi: 10.1016/j.gene.2016. 09.014

Zhao, J., Sinclair, J., Houghton, J., Bolton, E., Bradley, A., and Lever, A. (2010). Cytomegalovirus $\beta 2.7$ RNA transcript protects endothelial cells against apoptosis during ischemia/reperfusion injury. J. Heart Lung Transplant. 29, 342-345. doi: 10.1016/j.healun.2009.09.006

Conflict of Interest Statement: The authors declare that the research was conducted in the absence of any commercial or financial relationships that could be construed as a potential conflict of interest.

Copyright (c) 2017 Liu and Ding. This is an open-access article distributed under the terms of the Creative Commons Attribution License (CC BY). The use, distribution or reproduction in other forums is permitted, provided the original author(s) or licensor are credited and that the original publication in this journal is cited, in accordance with accepted academic practice. No use, distribution or reproduction is permitted which does not comply with these terms. 